\title{
The 7/5-Conjecture Strengthens Itself
}

\author{
A. V. Kostochka \\ INSTITUTE OF MATHEMATICS \\ SIBERIAN BRANCH \\ RUSSIAN ACADEMY OF SCIENCES \\ NOVOSIBIRSK, RUSSIA
}

\section{ABSTRACT}

The following is proved: if every bridgeless graph $G$ has a cycle cover of length at most $7 / 5|E(G)|$, then every bridgeless graph $G$ has a cycle cover of length at most $7 / 5|E(G)|$ such that any edge of $G$ is covered once or twice. (c) 1995 John Wiley \& Sons, Inc.

\section{INTRODUCTION}

A cycle cover $C$ of a graph $G=(V, E)$ is a collection of cycles $\left\{C_{1}, \ldots, C_{t}\right\}$ covering $E$. Its length is $l(C)=\left|C_{1}\right|+\cdots+\left|C_{t}\right|$. The cycle covering ratio $r(G)$ of graph $G=(V, E)$ with no cutedges is defined as the minimum of $l(C) /|E|$ over all the cycle coverings $C$ of $G$. It was remarked $[1,2,3]$ that there were many 2 -edge-connected graphs with covering ratio equal to $7 / 5$. There exists a folklore conjecture that

$$
r(G) \leq 7 / 5 \text { for any 2-edge-connected graph } G \text {. }
$$

Call a cycle covering $C$ of $G \operatorname{good}$ if any edge of $G$ is covered by at most two cycles. The well-known Cycle Double Cover $(C D C)$ Conjecture is equivalent to the statement that any 2-edge-connected graph has a good covering. U. Jamshy and M. Tarsi [4] showed that the validity of the 7/5-conjecture above implies the $\mathrm{CDC}$-conjecture. Here we use their approach to show the following:

Theorem. The $7 / 5$-conjecture implies that for any 2-edge-connected graph $G$ there exists a good cycle covering of $G$ of length at most $7 / 5|E(G)|$.

Journal of Graph Theory, Vol. 19, No. 1, 65-67 (1995)

(C) 1995 John Wiley \& Sons, Inc. 


\section{PROOF OF THE THEOREM}

Let $c(G)$ (respectively, $p(G)$ ) denote the length of the shortest cycle covering (respectively, the length of the shortest postman tour) of $G$. The following fact is obvious.

Observation. If $c(G) \leq p(G)+1$, then any cycle covering of $E(G)$ of length $c(G)$ is a good covering.

The observation may be applied to the Peterson graph $P$ since $c(P)=$ $21=1+p(P)$. Denote by $k P$ the result of replacing every edge of $P$ by a path of length $k$. Let $e=(x, y)$ be an edge in $k P$. Denote by $R_{k}$ the graph obtained from $k P$ by deleting $e$ and adding two vertices $x^{\prime}$ and $y^{\prime}$ and two edges $\left(x, x^{\prime}\right)$ and $\left(y, y^{\prime}\right)$. Then $R_{k}$ has $15 k-3$ vertices and $15 k+1$ edges.

Now, let $G$ be an arbitrary 2-edge-connected graph and $k=2|E(G)|$. Our aim is to prove that there exists a good cycle covering of $G$ of length at most $7 k / 10$ provided the $7 / 5$-conjecture is true. Construct the graph $H$ from $G$ by replacing each edge $(a, b)$ of $G$ by a copy $R_{k}(a, b)$ of $R_{k}$ so that $x^{\prime}$ coincides with $a$ and $y^{\prime}$ coincides with $b$. Then $H$ has $|V(G)|+(15 k-5)|E(G)|$ vertices and $|E(G)|(15 k+1)=k(15 k+1) / 2$ edges. By the $7 / 5$-conjecture, there is a cycle covering $C=\left\{C_{1}, \ldots, C_{t}\right\}$ of $H$ of length at most $7 k(15 k+1) / 10$. Remark that for any copy $R_{k}$ the edges $\left(x, x^{\prime}\right)$ and $\left(y, y^{\prime}\right)$ belong to the same cycles of $C$. This implies that

(1) $C$ induces a cycle covering $C(a, b)$ on the copy of $k P$ from which $R_{k}(a, b)$ was obtained, and

(2) $C$ induces a cycle covering $C(G)=\left\{C_{1}^{\prime}, \ldots, C_{t}^{\prime}\right\}$ of $G$ in the following way: $(a, b) \in C_{i}^{\prime}$ if and only if the edge $\left(x^{\prime}, x\right)$ in $R_{k}(a, b)$ belongs to $C_{i}$.

Since $c(P)=21$, the length of $C(a, b)$ is at least $21 k$, and, in view of the Observation, if $C(a, b)$ is not a good covering then its length is at least $22 k$. Hence assuming that $C$ is not good, we deduce that

$$
\begin{aligned}
l(C) & \geq 21 k(|E(G)|-1)+22 k=21 k(k / 2-1+22 / 21) \\
& =10.5 k(k+2 / 21)>(7 / 5) k(15 k+1) / 2=(7 / 5)|E(H)|,
\end{aligned}
$$

a contradiction. Thus, $C$ is a good covering. For every copy $R_{k}(a, b)$, we may consider the edge $\left(x, x^{\prime}\right)$ as belonging to $G$ and the edge $\left(y, y^{\prime}\right)$ as the edge completing $R_{k}(a, b)$ to $R_{k}$. As remarked, $l(C(a, b)) \geq 21 k$ for each $(a, b) \in E(G)$ and so, to cover all the edges of $H$ except the edges of the kind $\left(x, x^{\prime}\right)$ we spend at least $21 k^{2} / 2$ edges of cycles of $C$. Hence the length 
of the covering $C(G)$ is equal to

$$
\begin{aligned}
l(C)-\sum_{(a, b) \in E(G)} l(C(a, b)) & \leq 7 k(15 k+1) / 10-21 k^{2} / 2 \\
& \leq 7 / 10 k=7 / 5|E(G)|
\end{aligned}
$$

and this covering is good, as $C$ is good.

\section{DISCUSSION}

Conjecture. For any 2-edge-connected graph $G$ there exists a good cycle covering of $G$ of length at most $|E(G)|+|V(G)|-1$.

It can be shown that the minimal counterexample to the conjecture is 3-connected and has no 3- and 4-cycles.

\section{ACKNOWLEDGMENT}

The author thanks H. Fleischner, the host of the Workshop on CDC Conjecture (Vienna, 1991), during which this article was carried out. This work was partly supported by a grant within the program "Universities of Russia" and by Grant 93-011-1486 of the Russian Foundation of Fundamental Research.

\section{References}

[1] N. Alon and M. Tarsi, Covering multigraphs by simple circuits. SIAM J. Alg. Discrete Methods 6 (1985) 345-350.

[2] J. C. Bermond, B. Jackson, and F. Jaeger, Shortest covering of graphs with cycles. J. Combinat. Theory B 35 (1983) 297-308.

[3] A. Itai, R. J. Lipton, C. H. Papadimitriou, and M. Rodeh, Covering graphs by simple circuits. SIAM J. Comput. 10 (1981) 746-750.

[4] U. Jamshy and M. Tarsi, Short cycle covers and the Cycle Double Cover Conjecture. J. Combinat. Theory B 56 (1992) 197-204. 